ISSN 2079-9292

www.mdpi.com/journal/electronics

Article

\title{
A Theoretical Study on the Operation Principle of Hybrid Solar Cells
}

\section{Monishka Narayan and Jai Singh *}

School of Engineering and IT, Charles Darwin University, Darwin NT 0909, Australia; E-Mail: monishkarita.narayan@cdu.edu.au

* Author to whom correspondence should be addressed; E-Mail: jai.singh@cdu.edu.au; Tel.: +61-889-466-811; Fax: +61-889-466-366.

Academic Editor: Mohan Jacob

Received: 13 February 2015 / Accepted: 25 May 2015 / Published: 29 May 2015

\begin{abstract}
In this work, the operation mechanism of hybrid solar cells is studied explicitly. The excitation, diffusion and dissociation of singlet and triplet excitons and charge transport of free charge carriers are studied and their corresponding rates are calculated for a flexible P3HT:SiNW hybrid solar cell. The rates are found to be faster for singlet than triplet excitons. Possible loss mechanisms in hybrid solar cells have also been highlighted.
\end{abstract}

Keywords: hybrid solar cells; excitation; diffusion; dissociation

\section{Introduction}

The concept of hybrid solar cells was developed with an intent of combining the advantages of both organic and inorganic materials, where the organic acceptor layer was replaced with an inorganic material for enhanced absorption and stability while maintaining the bulk-heterojunction concept $[1,2]$. Hybrid materials normally consist of inorganic nanoparticles as acceptor [3] and organic materials (small molecules/polymers) as donor materials. Together they form the photovoltaic active layer where photon absorption, Frenkel exciton formation [4-6], diffusion [4] and dissociation [7,8] take place. The free charge carriers are transported and collected at opposite electrodes [2,7,9].

In hybrid solar cells, a combination of poly(3,4-ethylenedioxythiophene) poly(styrenesulfonate) (PEDOT:PSS) and silicon nanowires (SiNWs) has produced the best power conversion efficiency of $8.40 \%$ [10]. Other examples of inorganic acceptors include titanium dioxide [11], zinc oxide [12] and 
cadmium selenide nanocrystals [13]. The advantages of hybrid solar cells include the enhanced photoconductivity and higher absorption coefficient of inorganic nanoparticles than organic materials. Also the $\mathrm{n}$ and $\mathrm{p}$ types doping levels of nanoparticles can be varied easily during synthesis [3]. However, the power conversion efficiency and stability of hybrid solar cells are still lower than bulk-heterojunction organic solar cells. This may be attributed to the poor charge transport through the polymer-nanoparticle interface [3]. In this work, the operation mechanism of hybrid solar cells is studied explicitly. The rates of excitation, diffusion and dissociation of excitons are calculated and simultaneous formation and transport of free charge carriers are studied.

\section{Operation Mechanism in Hybrid Solar Cells}

In this section, the rates of exciton formation, diffusion and dissociation are studied using their corresponding interaction operators, transition matrix element and Fermi's Golden rule for hybrid solar cells.

\subsection{Excitation of Singlet and Triplet Excitons}

Absorption of light of energy larger than or equal to the bandgap of the donor organic solid results in the production of excited electron $(e)$ and hole $(h)$ pairs. An electron is excited to the lowest unoccupied molecular orbital, $\left(E_{L U M O}\right)$, leaving a hole in the highest occupied molecular orbital, $\left(E_{\text {номо }}\right.$, which instantly form an exciton due to strong Coulomb interaction between $e$ and $h$ caused by the low dielectric constant $(\varepsilon=3-4)$ of organic solids. As the electronic intermolecular interaction is weak in organics, the formation of such excitons is usually of Frenkel type [4,14-16]. The rate of a photon absorption for a singlet excitation due to exciton-photon interaction, $R_{a}^{S}$, and triplet excitation due to exciton-spin-orbit-photon interaction, $R_{a}^{T}$, are derived using the transition matrix element and Fermi's Golden rule and are given as [6]:

$$
\begin{gathered}
R_{a}^{S}=\frac{4 k e^{2}\left(E_{L U M O}-E_{\text {HOMO }}\right)^{3} a_{x s}^{2}}{3 c^{3} \varepsilon^{1.5} \hbar^{4}} \\
R_{a}^{T}=\frac{32 e^{6} Z^{2} k^{2} \varepsilon\left(E_{L U M O}-E_{\text {HOMO }}\right)}{c^{7} \varepsilon_{o} \mu_{x}^{4} a_{x t}^{4}}
\end{gathered}
$$

where $S$ denotes singlet and $T$ triplet, $k=\left(4 \pi \varepsilon_{o}\right)^{-1}=9 \times 10^{9}, e=$ electronic charge, $c=$ speed of light, $\varepsilon=$ dielectric constant, $\hbar=$ reduced Planck's constant, $Z=$ atomic number of heavy metal atom, $\varepsilon_{o}$ is the vacuum permittivity and $\mu_{x}$ is the reduced mass of exciton. The excitonic Bohr radius for singlet and triplet excitons $a_{x s}$ and $a_{x t}$, respectively, are given as [4]:

$$
\begin{gathered}
a_{x s}=\frac{\alpha^{2}}{(\alpha-1)^{2}} \frac{\mu \varepsilon}{\mu_{x}} a_{o} \\
a_{x t}=\frac{\mu \varepsilon}{\mu_{x}} a_{o}
\end{gathered}
$$

where $\alpha$ is a material dependent constant representing the ratio of the magnitude of the Coulomb and exchange interactions between the electron and hole in an exciton, $\mu_{x}$ is the reduced mass of an exciton, $a_{o}=5.29 \times 10^{-11} \mathrm{~m}$ is the Bohr radius and $\mu$ is the reduced mass of electron in a hydrogen atom. 


\subsection{Exciton Diffusion to Organic-Inorganic Interface}

After its formation, the exciton diffuses to the organic-inorganic interface. The two common exciton transfer mechanisms between the donor and acceptor materials are obtained through the Förster and Dexter transfer theories. They describe the transfer process of excitons as a function of the distance between the donor and acceptor materials [17]. Diffusion coefficient, $D$, can be determined for singlet and triplet excitons using the Förster and Dexter transfer, respectively, as [18]:

$$
\begin{gathered}
D_{S}=\frac{R_{f}{ }^{6}}{6 \tau R_{d a}{ }^{4}} \\
D_{T}=\frac{R_{d}^{2}}{6 \tau} \exp \left[\frac{2 R_{d}}{L}\left(1-\frac{R_{d a}}{R_{d}}\right)\right]
\end{gathered}
$$

where $R_{f}=$ Förster radius that determines the actual distance between the donor and acceptor, $R_{d a}=$ donor-acceptor separation distance and $\tau$ is the lifetime, $L$ is the average length of a molecular orbital and $R_{\mathrm{d}}$ is the Dexter radius between donor and acceptor at which the efficiency of such transfer remains at $50 \%$ [17]. The diffusion length, $L_{D}=\sqrt{D \tau}$, for singlet, and triplet excitons are obtained as [4]:

$$
\begin{gathered}
L_{D}^{S}=\frac{1}{\sqrt{6}} \frac{R_{f}{ }^{3}}{R_{d a}{ }^{2}} \\
L_{D}^{T}=\frac{R_{d}}{\sqrt{6}} \exp \left[\frac{R_{d}}{L}\left(1-\frac{R_{d a}}{R_{d}}\right)\right]
\end{gathered}
$$

\subsection{Exciton Dissociation at the Organic-Inorganic Interface}

After diffusion to the interface, the Frenkel exciton must dissociate into free charge carriers. The mechanism of exciton dissociation has been proposed in our previous study [7] and occurs through two steps: (1) As the energy of acceptor's conduction band energy level, $E_{C B}^{A}$, is lower than that of donor's LUMO, $E_{L U M O}^{D}$, a Frenkel exciton relaxes to a charge transfer (CT) exciton state where electron gets transferred to the acceptor's CB [19]. It is to be noted that the CT exciton is not yet dissociated because for dissociation it requires an external energy at least equal to the binding energy [20]. (2) This external energy comes from the excess vibrational energy released due to the formation of CT exciton. This excess vibrational energy, if adequate, may impact back to the CT exciton causing it to dissociate into free charge carriers. Free electrons, thus generated are drawn towards the cathode and holes towards the anode due to the potential energy difference caused by the difference in work function of the two electrodes [21,22].

The rate of exciton dissociation, $R_{\mathrm{d}}$, for singlet and triplet excitons in hybrid OSCs is derived using the interaction operator between $\mathrm{CT}$ exciton and molecular vibration energy, transition matrix element and Fermi's Golden rule and can be written as [7]:

$$
R_{d}^{S}=\frac{8 \pi^{2}}{3 \hbar^{3} \varepsilon^{2} E_{B}^{S}}\left[\left(E_{L U M O}^{D}-E_{C B}^{A}\right)-E_{B}^{S}\right]^{2}\left(\hbar \omega_{v}\right) \mu_{x} a_{x s}^{2}
$$




$$
R_{d}^{T}=\frac{8 \pi^{2}}{3 \hbar^{3} \varepsilon^{2} E_{B}^{T}}\left[\left(E_{L U M O}^{D}-E_{C B}^{A}\right)-E_{B}^{T}\right]^{2}\left(\hbar \omega_{v}\right) \mu_{x} a_{x t}^{2}
$$

where $\omega_{v}$ is the phonon frequency and $E_{B}^{S}$ and $E_{B}^{T}$ are the singlet and triplet exciton binding energies, respectively, given as [4]:

$$
\begin{gathered}
E_{B}^{S}=-\frac{(\alpha-1)^{2} \mu_{x} e^{4} k^{2}}{\alpha^{2} 2 \hbar^{2} \varepsilon^{2}} \\
E(S=1)=-\frac{\mu_{x} e^{4} k^{2}}{2 \hbar^{2} \varepsilon^{2}}
\end{gathered}
$$

After exciton dissociation, the free electron is transported to cathode and hole to anode with the aid of the electric field generated due to the workfunction difference between the electrodes and a force, $F$, of [7]:

$$
F=\frac{\Phi_{a}-\Phi_{c}}{r}
$$

where $\Phi_{a}$ and $\Phi_{c}$ are the work functions of the anode and cathode, respectively and $r$ is the inter-electrode distance.

\section{Results and Discussion}

In this section, the various rates are calculated using Equations (1)-(8) for a hybrid solar cell of the following structure: PET/PEDOT:PSS/TFB/P3HT:SiNW/Ca where polyethylene terephthalate $(\mathrm{PET})=$ the plastic substrate, PEDOT:PSS $=$ anode, poly[9,9-dioctylfluorene-co- $N$-[4-(3methylpropy1)]-diphenylamine] (TFB) = hole transport layer, polythiophene (P3HT) as donor: SiNW as acceptor and Calcium $(\mathrm{Ca})=$ anode $[9,23,24]$. P3HT is a wide band gap polymer and is the most popular and studied donor material used in organic photovoltaics [25,26] and SiNWs ensure large donor-acceptor intefacial area with continous interpenetrating pathways for better charge transport to the electrodes [27]. The input parameters for calculations are as follows: $a_{x s}=4.35 \mathrm{~nm}$ and $a_{x t}=0.32 \mathrm{~nm}$, $\mathrm{Z}=77$ for Iridium, $\varepsilon=7, \mu_{x}=1.99 \times 10^{-26} \mathrm{~kg}, E_{B}^{S}=0.06 \mathrm{eV}, E_{B}^{T}=0.76 \mathrm{eV}$ [4], $R_{d a}=0.05 \mathrm{~nm}$, $R_{f}=8 \mathrm{~nm}, \tau=2230 \mathrm{ps}$ [28], $L=0.11 \mathrm{~nm}, R_{d}=1 \mathrm{~nm} \mathrm{[29]} E_{L U M O}^{P 3 H T}=-3.03 \mathrm{eV}, E_{C B}^{S i N W}=-4.00 \mathrm{eV} \mathrm{[30].}$ Using the above design and parameters, the various rates are calculated as shown in Table 1 . These rates depend on the above input parameters and therefore some uncertainty may be expected due to the uncertainties in the parameters themselves. It is not possible exactly to estimate variations in the input parameters however according to Equations (3), (4), (11) and (12), singlet and triplet Bohr radii and corresponding binding energies depend on the parameters $\alpha$ and $\mu_{x}$ which are estimated to be equal to 1.37 and $0.5 m_{e}$, respectively [4]. The estimate of the effective mass of charge carriers in organic materials to be equal to the electronic rest mass $m_{e}$ is regarded to be a good approximation as the HOMO and LUMO levels are electronic molecular states instead of energy bands. The parameter $\alpha$ is estimated from the measured value of the exchange energy $\Delta E=0.7 \mathrm{eV}$ [31] between singlet and triplet excitons, which is also a reasonable approximation. However, according to Equations (9) and (10), the rates of dissociation of excitons depend on the squires of both the exciton binding energy and exciton Bohr radius. This means that any uncertainty in these quantities may be expected to get squared in the rates. 
Table 1. Rates of excitation, diffusion and dissociation in PET/PEDOT:PSS/TFB/ P3HT:SiNW/Ca hybrid solar cell.

\begin{tabular}{ccc}
\hline Mechanism & Parameter & Value \\
\hline \multirow{2}{*}{ Excitation } & $R_{a}^{S}$ & $1.27 \times 10^{10} \mathrm{~s}^{-1}$ \\
& $R_{a}^{T}$ & $3.09 \times 10^{5} \mathrm{~s}^{-1}$ \\
\hline \multirow{3}{*}{ Diffusion } & $D_{S}$ & $1.99 \mathrm{~cm}^{2} \mathrm{~s}^{-1}$ \\
& $D_{T}$ & $2.71 \times 10^{-6} \mathrm{~cm}^{2} \cdot \mathrm{s}^{-1}$ \\
& $L_{D}^{S}$ & $2.82 \times 10^{2} \mathrm{~nm}$ \\
& $L_{D}^{T}$ & $1.17 \mathrm{~nm}$ \\
\hline \multirow{2}{*}{ Dissociation } & $R_{d}^{S}$ & $2.60 \times 10^{17} \mathrm{~s}^{-1}$ \\
& $R_{d}^{T}$ & $2.98 \times 10^{12} \mathrm{~s}^{-1}$ \\
\hline \multirow{2}{*}{ Charge transport } & $F$ & $3.36 \times 10^{-12} \mathrm{~N}$ \\
\hline
\end{tabular}

The energies of LUMO and HOMO of P3HT and that of CB of SiNW are taken from Ref. [30], where the choice is based on the following experimental conditions: The HOMO of P3HT should be positioned above the valence band (VB) of SiNW to inject holes into the hole transport layer and hence into the anode and at the same time it should also accept holes generated by light absorption in the SiNW. The LUMO of P3HT should be located above the CB of SiNW so that electron collection can occur efficiently at the silicon interface and electrons generated in the nanowires can be collected at the cathode [30].

According to Table 1, the rate of excitation of singlet excitons is four orders of magnitude greater than that of triplet excitons in P3HT:SiNW hybrid solar cell. This is because $R_{a}^{S}$ is highly sensitive to the absorption energy, $\left(E_{L U M O}-E_{\text {HOMO }}\right)^{3}$ while $R_{a}^{T}$ is only linearly dependent on $\left(E_{L U M O}-E_{\text {HOMO }}\right)$. The triplet excitation rate is facilitated by the incorporation of heavy metal atom, Iridium, due to enhanced exciton-spin-orbit-photon interaction which also flips the spin to singlet configuration to facilitate the absorption by spin conservation. At the same time, flipped spins to singlet configuration makes it easier to dissociate the exciton into free charge carriers due to their low binding energy [4].

After their formation, the exciton diffuses to the P3HT:SiNW interface. Due to the bulk nature of the hybrid solar cell, the heterojunction is distributed throughout the layer. This enables charge carrier generation everywhere within the active layer [32]. The singlet diffusion coefficient is about five orders of magnitude greater than the triplet, however, the lifetime of triplet is longer than singlet exciton. According to Table 1, the singlet diffusion length is two orders of magnitude greater than triplet exciton.

After successfully reaching the P3HT:SiNW interface, the Frenkel exciton relaxes to CT exciton and simultaneously dissociates into free charge carriers due to the excess vibrational energy released due to the offset i.e., $E_{L U M O}^{P 3 H T}-E_{C B}^{S i N W}=0.80 \mathrm{eV}$ [9]. At such offset, dissociation of both singlet and triplet excitons is ensured because it surpasses their binding energies, however, the singlet dissociation rate is five orders of magnitude faster than that of triplet. Both the dissociation rates are higher than the corresponding recombination rates which implies that the dissociation of an exciton may be faster than its non-radiative recombination, which enhances the performance of the hybrid solar cells [6,7].

The dissociated free electrons and holes are collected by the work function difference between the electrodes i.e., $\Phi_{\text {PEDOT.PSS }}-\Phi_{C a}=2.10 \mathrm{eV}$, which creates a reasonably large built-in field for 
efficient transport and collection of holes at PEDOT:PSS and electrons at Ca. A force of $3.36 \mathrm{pN}$ is available for charge transport as shown in Table 1. Also, in our previous study, we have optimzed the design of P3HT:SiNW hybrid solar cell using the semiconducting thin film optics simulation software and a power conversion efficiency of $4.70 \%$ was obtained [9]. This is an enhancement of $>50 \%$ from its current experimental performance [33].

Loss mechanisms also occur due to the inefficient transfer of Frenkel exciton to P3HT:SiNW interface as the probability of an exciton created at a molecule to move to any one particular direction is $1 / 4 \pi$. If this molecule is next to the interface then the transfer should be multiplied by $1 / 4 \pi$. However, if this interface is a few molecules away from the excited molecule, the rate of transfer should be multiplied by $1 / 4 \pi$ at each such molecular step. Thus, the probability that an exciton created $n$ molecules away from the interface will reach the interface becomes $(1 / 4 \pi)^{n}$. As $4 \pi \sim 10$, the further the exciton is from the interface, much less is the probability that it will reach the interface to form a CT exciton. This basically means that every single step away from the interface will reduce the probability by at least one order of magnitude [7].

Also, the vibrational energy can be dissipated in any direction from the excited molecule. The probability that it will be transferred back to the CT exciton to dissociate it is only one of the possible directions of a solid angle. This means that the rate of dissociation reduces by a factor of $(1 / 4 \pi)^{2}$, which is nearly three orders of magnitude less than that obtained from Equations (6) and (7) and hence the dissociation time will be longer by three orders of magnitude.

Also, in our previous study, it has been found that considering the charge transport in P3HT:SiNW hybrid OSC, the electron mobility is much higher than the hole mobility, $\mu_{e}>\mu_{h}$ i.e., electron will reach the $\mathrm{Ca}$ electrode much faster than the hole can reach PEDOT:PSS. This leads to hole accumulation within the bulk which may lead to non-radiative quenching of holes and a low free carrier collection efficiency is obtained. However, the results presented in this study may be expected to assist in understanding the operation and loss mechanisms in hybrid solar cells.

\section{Conclusions}

The operation mechanism in hybrid solar cells has been studied with respect to exciton formation, diffusion and dissociation. It is found that in a P3HT:SiNW hybrid solar cell, rates of singlet excitons, diffusion and dissociation are higher compared to those of triplet excitons. The diffusion length of singlet excitons is found to be higher than that of triplet excitons which can be attributed to the larger diffusion coefficient of singlet excitons than that of triplet excitons. The offset at the organic-inorganic interface ensures efficient dissociation of singlet and triplet excitons, however, the mismatch in electron and hole mobilities may affect the charge transport in P3HT:SiNW hybrid solar cells.

\section{Author Contributions}

Monishka Narayan carried out all the calculations as a Ph.D. student of Jai Singh who has supervised her work all through her Ph.D. candidature. With the guidance from Singh, the paper was first drafted by Narayan and then was finalized by them together. 


\section{Conflicts of Interest}

The authors declare no conflict of interest.

\section{References}

1. Wright, M.; Uddin, A. Organic-Inorganic hybrid solar cells: A comparative review. Sol. Energy Mater. Sol. Cells 2012, 107, 87-111.

2. Chandrasekaran, J.; Nithyaprakash, D.; Ajjan, K.B.; Maruthamuthu, S.; Manoharan, D.; Kumar, S. Hybrid solar cell based on blending of organic and inorganic materials-An overview. Renew. Sustain. Energy Rev. 2011, 15, 1228-1238.

3. Arici, E.; Sariciftci, N.S.; Meissner, D. Hybrid solar cells. Encycl. Nanosci. Nanotechnol. 2004, 3, 929-944.

4. Narayan, M.R.; Singh, J. Roles of binding energy and diffusion length of singlet and triplet excitons in organic heterojunction solar cells. Phys. Status Solidi 2012, 9, 2386-2389.

5. Narayan, M.R.; Singh, J. Effect of exciton-spin-orbit-photon interaction in the performance of organic solar cells. Eur. Phys. J. B 2013, 86, 1-4.

6. Narayan, M.R.; Singh, J. Effect of simultaneous excitation of singlet and triplet excitons on the operation of organic solar cells. J. Appl. Phys. 2013, 114, 154515-154517.

7. Narayan, M.R.; Singh, J. Study of the mechanism and rate of exciton dissociation at the donor-acceptor interface in bulk-heterojunction organic solar cells. J. Appl. Phys. 2013, 114, 073510-073517.

8. Narayan, M.R.; Singh, J. Exciton dissociation and design optimization in p3ht:Pcbm bulk-heterojunction organic solar cell. Can. J. Phys. 2013, 92, 853-856.

9. Narayan, M.R.; Singh, J. Optimizing the design of flexible ptb7:Pc71bm bulk-heterojunction and p3ht:Sinw hybrid organic solar cells. J. Nanosci. Nanotechnol. 2013, 1, 8.

10. Syu, H.; Shiu, S.; Lin, C. Silicon nanowire/organic hybrid solar cell with efficiency of $8.40 \%$. Sol. Energy Mater. Sol. Cells 2012, 98, 267-272.

11. Plass, R.; Pelet, S.; Krueger, J.; Grätzel, M.; Bach, U. Quantum dot sensitization of organic-inorganic hybrid solar cells. J. Phys. Chem. B 2002, 106, 7578-7580.

12. Beek, W.J.; Wienk, M.M.; Janssen, R.A. Efficient hybrid solar cells from zinc oxide nanoparticles and a conjugated polymer. Adv. Mater. 2004, 16, 1009-1013.

13. Huynh, W.U.; Dittmer, J.J.; Alivisatos, A.P. Hybrid nanorod-polymer solar cells. Science 2002, 295, 2425-2427.

14. Roncali, J. Molecular bulk heterojunctions: An emerging approach to organic solar cells. Acc. Chem. Res. 2009, 42, 1719-1730.

15. Kippelen, B.; Brédas, J.L. Organic photovoltaics. Energy Environ. Sci. 2009, 2, 251-261.

16. Brédas, J.L.; Norton, J.E.; Cornil, J.; Coropceanu, V. Molecular understanding of organic solar cells: The challenges. Acc. Chem. Res. 2009, 42, 1691-1699.

17. Steinbacher, F.S.; Krause, R.; Hunze, A.; Winnacker, A. Triplet exciton transfer mechanism between phosphorescent organic dye molecules. Phys. Status Solidi 2012, 209, 340-346. 
18. Lunt, R.R.; Giebink, N.C.; Belak, A.A.; Benziger, J.B.; Forrest, S.R. Exciton diffusion lengths of organic semiconductor thin films measured by spectrally resolved photoluminescence quenching. J. Appl. Phys. 2009, 105, 053711-053717.

19. Zhu, X.-Y.; Yang, Q.; Muntwiler, M. Charge-transfer excitons at organic semiconductor surfaces and interfaces. Acc. Chem. Res. 2009, 42, 1779-1787.

20. Scharber, M.C.; Mühlbacher, D.; Koppe, M.; Denk, P.; Waldauf, C.; Heeger, A.J.; Brabec, C.J. Design rules for donors in bulk-heterojunction solar cells-Towards $10 \%$ energy-conversion efficiency. Adv. Mater. 2006, 18, 789-794.

21. Kendrick, M.J.; Neunzert, A.; Payne, M.M.; Purushothaman, B.; Rose, B.D.; Anthony, J.E.; Haley, M.M.; Ostroverkhova, O. Formation of the donor-acceptor charge transfer exciton and its contribution to charge photogeneration and recombination in small-molecule bulk heterojunctions. J. Phys. Chem. C 2012, 116, 18108-18116.

22. Bruder, I. Organic Solar Cells: Correlation between Molecular Structure, Morphology and Device Performance; Max-Planck-Institut für Festkörperforschung, Stuttgart, Germany, 2010.

23. Davenas, J.; Dkhil, S.B.; Cornu, D.; Rybak, A. Silicon nanowire/p3ht hybrid solar cells: Effect of the electron localization at wire nanodiameters. Energy Proced. 2012, 31, 136-143.

24. Liu, K.; Qu, S.; Zhang, X.; Tan, F.; Wang, Z. Improved photovoltaic performance of silicon nanowire/ organic hybrid solar cells by incorporating silver nanoparticles. Nanoscale Res. Lett. 2013, 8, 1-6.

25. Dang, M.T.; Hirsch, L.; Wantz, G. P3ht: Pcbm, best seller in polymer photovoltaic research. Adv. Mater. 2011, 23, 3597-3602.

26. Chen, D.; Nakahara, A.; Wei, D.; Nordlund, D.; Russell, T.P. P3ht/pcbm bulk heterojunction organic photovoltaics: Correlating efficiency and morphology. Nano Lett. 2010, 11, 561-567.

27. Kuo, C.Y.; Gau, C. Arrangement of band structure for organic-inorganic photovoltaics embedded with silicon nanowire arrays grown on indium tin oxide glass. Appl. Phys. Lett. 2009, 95, 053302.

28. Huang, J.-S.; Goh, T.; Li, X.; Sfeir, M.Y.; Bielinski, E.A.; Tomasulo, S.; Lee, M.L.; Hazari, N.; Taylor, A.D. Polymer bulk heterojunction solar cells employing forster resonance energy transfer. Nat. Photonics 2013, 7, 479-485.

29. Mangold, H. Charge Separation and Recombination in Novel Polymeric Absorber Materials for Organic Solar Cells-A Photophysical Study. Ph.D. Thesis, Johannes Gutenberg-Universität Mainz, Mainz, Germany, 2013.

30. Azizi, S.; Braik, M.; Dridi, C.; Ouada, H.B.; Ryback, A.; Davenas, J. Study of charge transport in p3ht: Sinw-based photovoltaic devices. Appl. Phys. A 2012, 108, 99-106.

31. Köhler, A.; Beljonne, D. The singlet-triplet exchange energy in conjugated polymers. Adv. Func. Mater. 2004, 14, 11-18.

32. Blom, P.W.M.; Mihailetchi, V.D.; Koster, L.J.A.; Markov, D.E. Device physics of polymer: Fullerene bulk heterojunction solar cells. Adv. Mater. 2007, 19, 1551-1566.

33. Huang, J.; Hsiao, C.; Syu, S.; Chao, J.; Lin, C. Well-aligned single-crystalline silicon nanowire hybrid solar cells on glass. Sol. Energy Mater. Sol. Cells 2009, 93, 621-624.

(C) 2015 by the authors; licensee MDPI, Basel, Switzerland. This article is an open access article distributed under the terms and conditions of the Creative Commons Attribution license (http://creativecommons.org/licenses/by/4.0/). 